February 26, 2019

\title{
CREMONA CONVEXITY, FRAME CONVEXITY, AND A THEOREM OF SANTALÓ
}

\author{
JACOB E. GOODMAN, ANDREAS HOLMSEN, RICHARD POLLACK, KRISTIAN RANESTAD, \\ AND FRANK SOTTILE
}

\begin{abstract}
In 1940, Luis Santaló proved a Helly-type theorem for line transversals to boxes in $\mathbb{R}^{d}$. An analysis of his proof reveals a convexity structure for ascending lines in $\mathbb{R}^{d}$ that is isomorphic to the ordinary notion of convexity in a convex subset of $\mathbb{R}^{2 d-2}$. This isomorphism is through a Cremona transformation on the Grassmannian of lines in $\mathbb{P}^{d}$, which enables a precise description of the convex hull and affine span of up to $d$ ascending lines: the lines in such an affine span turn out to be the rulings of certain classical determinantal varieties. Finally, we relate Cremona convexity to a new convexity structure that we call frame convexity, which extends to arbitrary-dimensional flats in $\mathbb{R}^{d}$.
\end{abstract}

\section{INTRODUCTION}

A cornerstone of the theory of convexity is Helly's theorem [10, which states that if there is a point common to every $d+1$ (or fewer) members of a collection of convex sets in $\mathbb{R}^{d}$, then the whole family has a point in common. Vincensini [15] asked if there was a similar result for line secants to convex sets in the plane. In 1940, Santaló showed that this was impossible in general, found a sufficient condition for the result to hold for lines in the plane, and generalized that to lines in $\mathbb{R}^{d}$, giving a Helly-type theorem for line transversals to boxes [13. A box in $\mathbb{R}^{d}$ is a product of $d$ intervals, one in each coordinate direction.

Theorem 1 (Santaló). If every $2^{d-1}(2 d-1)$ (or fewer) members of a collection of boxes in $\mathbb{R}^{d}$ admit a line transversal, then there is a line transversal to all the boxes.

This was the first of a great many results in what is now known as geometric transversal theory [2, 3, 6, 16, 17. Santaló's elementary proof was based on an idea that Radon had used to establish Helly's theorem [12. Grünbaum [8] showed that these numbers are the best possible; there are 6 squares in the plane, every five of which have a line transversal, but all six do not. If we ignore the factor $2^{d-1}$, which is simply the number of direction classes for lines in $\mathbb{R}^{d}$ that are not parallel to any coordinate hyperplane, the number in Santaló's theorem is the Helly number for dimension $2 d-2$, which is the dimension of the space of lines in $\mathbb{R}^{d}$. This is not a coincidence - for as we shall see, Santaló's theorem is essentially Helly's theorem in disguise.

2000 Mathematics Subject Classification. Primary: 52A35; Secondary: 14M15, 14M12.

Key words and phrases. Helly theorem, Convexity, Grassmannian, Cremona transformation.

Research of first author supported in part by NSA grant MDA904-03-I-0087 and PSC-CUNY grant 65440-0034; research of second author supported in part by the Mathematical Sciences Research Institute, Berkeley; research of third author supported in part by NSF grant CCR-9732101; research of fifth author supported in part by NSF CAREER grant DMS-0134860 and the Clay Mathematical Institute. 
A line in $\mathbb{R}^{d}$ is weakly ascending if all non-zero coordinates of its direction vector have the same sign. If no coordinates of its direction vector vanish, then such a line is ascending. A frame-convex set of (ascending) lines is the set of lines that meet every box in a given collection of boxes. (Since the notion of a 'box' is dependent on the choice of coordinate frame, this notion of convexity is as well.) This is related to a general theory of convexity on Grassmannians due to Goodman and Pollack [5] in which a set $\mathcal{K}$ of $k$-flats is convex if whenever a $k$-flat $K$ meets every convex body meeting every member of $\mathcal{K}$, then $K$ lies in $\mathcal{K}$. For frame convexity, we restrict the convex bodies to be boxes and work with lines rather than $k$-flats. Weakly ascending lines admit natural Cremona coordinates (described in Section 1) which identify them with a convex set in $\mathbb{R}^{2 d-2}$.

Theorem 2. The Cremona coordinates of a frame-convex set of weakly ascending lines form a convex set.

This elementary result can be seen in a close reading of Santaló's arguments. We give a short proof of Theorem 2 and also of Santaló's theorem in Section 11. The converse of Theorem 2 is not true in general. A set $S$ of weakly ascending lines is Cremona-convex if the set of Cremona coordinates of its members form a convex set. Suppose that $\ell$ and $\ell^{\prime}$ are ascending lines in $\mathbb{R}^{d}$ that meet in a point. Then their Cremona-convex hull is 1-dimensional, but their frame-convex hull may have any dimension between 1 and $d-1$. The case $d=3$ is described in Example 6. Despite this, these two notions of convexity coincide for ascending skew lines that satisfies the additional condition that the images of the direction vectors by the orthogonal projection to any coordinate plane are distinct (see Corollary 18).

For ascending lines, the Cremona coordinates of Theorem 2 come from a particular Cremona transformation on Plücker space that transforms the Grassmannian of lines into a linear space. This linearizing Cremona transformation is related to Kapranov's identification of the Chow quotient of the Grassmannian of lines as the space $M_{0, n}$ of $n$ marked points on $\mathbb{P}^{1}[11$, and it underlies some recent work on the tropical Grassmannian of lines [1, 14].

We obtain a precise geometric description of the Cremona-convex hull and Cremonaaffine span of a set of lines. For example, consider two skew ascending lines in $\mathbb{R}^{3}$ such that there is a unique line parallel to each axis meeting the two given lines. Then these three axis-parallel lines lie on a unique doubly ruled quadric, either a hyperboloid of one sheet or a hyperbolic paraboloid. The three axis-parallel lines lie in one ruling and the two original lines lie in the other. The two original lines determine two intervals in their ruling (three in the case of a hyperbolic paraboloid). One of those intervals consists solely of ascending lines, and this interval is the frame-convex interval between the original two lines. This is shown in the figure on the left in Figure 1. The Cremona-affine span of three general lines in $\mathbb{R}^{3}$ consists of the secant lines to a particular rational cubic curve. This is illustrated in the figure on the right in Figure 1, where we display two branches of the rational cubic and lines that are the extreme points of the Cremona-convex hull of three lines. In Section [2.2 we explain this more generally in $\mathbb{R}^{d}$.

Replacing boxes by parallelepipeds with parallel edges gives the coordinate-free version of frame convexity. This was the original context of Santaló's results. Santaló also proved a Helly-type theorem for hyperplane transversals, which follows from the observation that for ascending hyperplanes (suitably defined), frame convexity coincides with a natural notion of convexity given by the coefficients of the defining equation. 

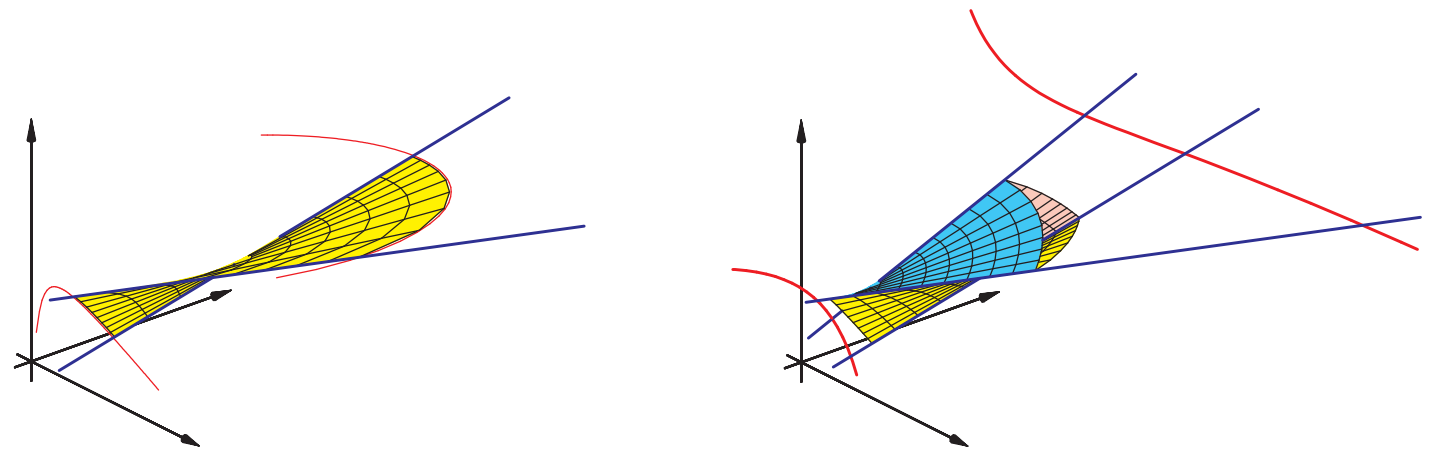

Figure 1. Cremona-convex hulls

Santaló asked if his results could be extended to $k$-flats in $\mathbb{R}^{d}$, for $1<k<d-1$. We do not know if this is possible, but feel that it is unlikely. In part that is because the linearizing Cremona transformation does not generalize to the Grassmannian with $1<k<d-2$. Also, we show that the frame-convex hull of two general 2-flats in $\mathbb{R}^{4}$ or $\mathbb{R}^{5}$ (suitably defined) is just the two original 2-flats, and thus frame convexity in this context has a completely different character than for lines or hyperplanes. On the other hand, since the set of $(d-2)$ flats in $\mathbb{P}^{d}$ is isomorphic to the set of lines in $\mathbb{P}^{d}$, the notion of frame convexity for ascending lines can be transferred to $(d-2)$-flats and gives it a convexity structure. We describe this in Section 3, and derive the corresponding theorem of Helly type.

We thank Bernd Sturmfels, who pointed out to us the relation of the Cremona transformation to Chow quotients and to the tropical Grassmannian.

\section{Convexity FOR WEAKLy ASCENDING LINES IN $\mathbb{R}^{d}$}

A weakly ascending line $\ell$ in $\mathbb{R}^{d}$ is determined by a point $x \in \ell$ and its direction vector $v$, which has non-negative coordinates. Call such a pair $(x, v)$ rectilinear coordinates for $\ell$. The Cremona coordinates $(y, w)$ for a line $\ell$ are defined as follows

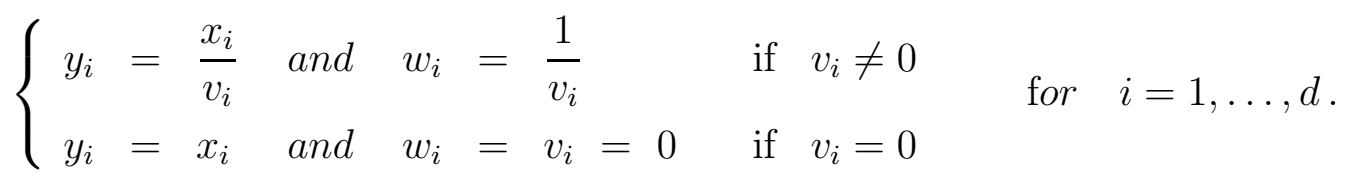

The first Cremona coordinate $y$ of a weakly ascending line is well-defined modulo translation by the vector $(1, \ldots, 1)$ and the second Cremona coordinate $w$ is well-defined modulo multiplication by a positive scalar. If we remove these ambiguities by requiring $\sum_{i} y_{i}=0$ and $\sum_{i} w_{i}=1$, then the Cremona coordinates take values in a convex subset of a $(2 d-2)$ dimensional affine subspace of $\mathbb{R}^{2 d}$.

Definition 3. A set of weakly ascending lines is Cremona-convex if its set of Cremona coordinates is convex.

These definitions imply that the dimension of the Cremona-convex hull of $k$ lines in $\mathbb{R}^{d}$ is at most $k-1$, with equality only if the Cremona coordinates are affinely independent. With this definition, Theorem 2 becomes the following. 
Theorem 2. A frame-convex set of weakly ascending lines is Cremona-convex.

Since an ascending line transversal to a collection of boxes is a common member of each of the frame-convex sets of ascending lines meeting each box, Helly's theorem applied to such convex sets gives a local version of Santaló's theorem.

Corollary 4. If every $2 d-1$ (or fewer) members of a collection of boxes in $\mathbb{R}^{d}$ admit an ascending line transversal, then there is an ascending line transversal to all the boxes.

Proof of Theorem 2. A box in $\mathbb{R}^{d}$ is the coordinatewise interval between its (coordinatewise) minimal and maximal points. The rectilinear coordinates $(x, v)$ of ascending lines that meet a box with minimal point $a$ and maximal point $b$ satisfy the inequalities

$$
\begin{gathered}
\max _{i: v_{i} \neq 0}\left\{\frac{a_{i}-x_{i}}{v_{i}}\right\} \leq \min _{i: v_{i} \neq 0}\left\{\frac{b_{i}-x_{i}}{v_{i}}\right\}, \quad \text { and } \\
a_{i} \leq x_{i} \leq b_{i} \quad \text { if } \quad v_{i}=0 .
\end{gathered}
$$

Indeed, a line with rectilinear coordinates $(x, v)$ meets the box if and only if the system of inequalities

$$
a_{i} \leq x_{i}+t \cdot v_{i} \leq b_{i} \quad \text { for } \quad i=1, \ldots, n
$$

has a solution in $t$, for then $x+t \cdot v$ lies in the box. The feasibility of this system for weakly ascending lines is expressed by (1.2).

In terms of Cremona coordinates, the inequalities (1.2) become

$$
\begin{array}{r}
\max _{i: w_{i} \neq 0}\left\{a_{i} w_{i}-y_{i}\right\} \leq \min _{i: w_{i} \neq 0}\left\{b_{i} w_{i}-y_{i}\right\}, \quad \text { and } \\
a_{i} \leq y_{i} \leq b_{i} \quad \text { if } \quad w_{i}=0
\end{array}
$$

which define a convex set.

Remark 5. Cremona coordinates of a line are the coefficients of some special equations for this line. (They appear in Santaló's proof in this form.) Let $z_{1}, \ldots, z_{d}$ be coordinates for $\mathbb{R}^{d}$, and consider a line $\ell$ with rectilinear coordinates $(x, v)$ where no coordinate of $v$ vanishes. Scaling if necessary, we may assume that $v_{1}=1$, and then transforming $x$ by $v$, that $x_{1}=0$. Then the line $\ell$ given by $x+t v$ is cut out by the linear forms

$$
v_{i} \cdot z_{1}-z_{i}+x_{i}=0, \quad \text { for } i=2, \ldots, d .
$$

If we divide by $v_{i}$, this becomes

$$
z_{1}-w_{i} \cdot z_{i}+y_{i}=0 \text { if } w_{i} \neq 0 \quad \text { for } i=2, \ldots, d .
$$

Santaló's proof uses a convex combination of the coefficients $\left(b_{i}, c_{i}\right)$ of the linear equations of a line that have the form

$$
a \cdot z_{1}+b_{i} \cdot z_{i}=c_{i} \quad i=2, \ldots, d,
$$

which we recognize from (1.3) as essentially the Cremona coordinates.

While Cremona coordinates of a line have this simple natural explanation as the coefficients of linear forms defining the line, that ad hoc realization belies their naturality as the coordinates for the Grassmannian in the linearizing Cremona transformation given in 
Section 2.1. Using this Cremona transformation, we will obtain a precise description of the Cremona-convex hull of a finite set of ascending lines.

Proof of Santalo's theorem. The closed orthants in the hyperplane at infinity are indexed by the signs of coordinates of points in their interiors. These vectors $\varepsilon \in\{ \pm 1\}^{d}$ are defined modulo multiplication by \pm 1 . A line $\ell$ has $\operatorname{sign} \varepsilon$ if it meets the hyperplane at infinity in the closed orthant corresponding to $\varepsilon$. Equivalently, if the numbers

$$
\varepsilon_{i} v_{i} \quad i=1, \ldots, d
$$

are either all non-negative or all non-positive, where $v$ is the direction vector of $\ell$.

Our previous notions and results hold, mutatis mutandis, for lines with a fixed sign. Every line has Cremona coordinates (1.1), and we call a set of lines with the same sign Cremona-convex if its corresponding set of Cremona coordinates is convex. The full set of lines with a given sign is Cremona-convex.

Corollary 4. If every $2 d-1$ (or fewer) members of a collection of boxes in $\mathbb{R}^{d}$ admit a line transversal with sign $\varepsilon$, then the collection has a line transversal with sign $\varepsilon$.

Suppose that we have a family of boxes such that every subset $S$ of size at most $2^{d-1}(2 d-1)$ admits a line transversal. Then there is a sign $\varepsilon$ such that every collection $S$ of $2 d-1$ boxes from our family admits a line transversal with $\operatorname{sign} \varepsilon$. Suppose this were not the case. Then for every $\operatorname{sign} \varepsilon$, there is a set $S_{\varepsilon}$ of $2 d-1$ boxes that does not admit a line transversal with $\operatorname{sign} \varepsilon$. But then the union of the sets $S_{\varepsilon}$ cannot admit a line transversal, which is a contradiction, as this union contains at most $2^{d-1}(2 d-1)$ boxes. Santaló's theorem now follows by its local version, Corollary 4 .

Example 6. Suppose that $\ell \neq \ell^{\prime}$ are ascending lines in $\mathbb{R}^{3}$ that meet in a point, which we assume is the origin. Let $(a, b, c)$ be the direction vector of $\ell$ and $\left(a^{\prime}, b^{\prime}, c^{\prime}\right)$ that for $\ell^{\prime}$. Then Cremona coordinates for $\ell$ and $\ell^{\prime}$ are

$$
\left[(0,0,0),\left(\frac{1}{a}, \frac{1}{b}, \frac{1}{c}\right)\right] \quad \text { and } \quad\left[(0,0,0),\left(\frac{1}{a^{\prime}}, \frac{1}{b^{\prime}}, \frac{1}{c^{\prime}}\right)\right],
$$

and their Cremona-convex hull consists of lines through the origin with direction vector

$$
\left(\frac{1}{\frac{p}{a}+\frac{p^{\prime}}{a^{\prime}}}, \frac{1}{\frac{p}{b}+\frac{p^{\prime}}{b^{\prime}}}, \frac{1}{\frac{p}{c}+\frac{p^{\prime}}{c^{\prime}}}\right), \quad \text { where } \quad p, p^{\prime} \geq 0 \quad \text { and } \quad p+p^{\prime}=1 .
$$

These direction vectors $(x, y, z)$ satisfy the quadratic equation

$$
\left|\begin{array}{ccc}
x y & x z & y z \\
b c & a c & a b \\
b^{\prime} c^{\prime} & a^{\prime} c^{\prime} & a^{\prime} b^{\prime}
\end{array}\right|=0
$$

where $|\cdot|$ denotes determinant.

This defines is an irreducible quadric unless one of the $2 \times 2$-minors involving the last two rows of the matrix (1.4) vanishes. When that happens, $\ell$ and $\ell^{\prime}$ span a plane that contains a coordinate axis. Otherwise, the Cremona-convex hull of $\ell$ and $\ell^{\prime}$ consists of some lines that rule the cone with apex the origin that is defined by the equation (1.4). Specifically, the 
lines $\ell$ and $\ell^{\prime}$ define two intervals in the ruling of this cone, one of which consists entirely of ascending lines, and this interval is their Cremona-convex hull.

The situation is different for the frame-convex hull of $\ell$ and $\ell^{\prime}$.

Proposition 7. Suppose that

$$
\left|\begin{array}{cc}
a & b \\
a^{\prime} & b^{\prime}
\end{array}\right| \cdot\left|\begin{array}{cc}
a & c \\
a^{\prime} & c^{\prime}
\end{array}\right| \leq 0
$$

Then the frame-convex hull of $\ell$ and $\ell^{\prime}$ is the set of ascending lines through the origin with direction vector $(\alpha, \beta, \gamma)$ satisfying

$$
\frac{b^{\prime}}{a^{\prime}} \leq \frac{\beta}{\alpha} \leq \frac{b}{a} \quad \text { and } \quad \frac{c}{a} \leq \frac{\gamma}{\alpha} \leq \frac{c^{\prime}}{a} .
$$

This is a 2-dimensional family of lines, except when one of the determinants (1.5) vanishes, and then it is one-dimensional. Since $\ell \neq \ell^{\prime}$, at most one determinant will vanish.

Remark 8. The assumption of Proposition 7 can always be satisfied by permuting the coordinate directions. Indeed, we have that

$$
\text { either } \quad\left|\begin{array}{cc}
a & b \\
a^{\prime} & b^{\prime}
\end{array}\right| \cdot\left|\begin{array}{cc}
a & c \\
a^{\prime} & c^{\prime}
\end{array}\right| \leq 0 \quad \text { or } \quad\left|\begin{array}{cc}
a & b \\
a^{\prime} & b^{\prime}
\end{array}\right| \cdot\left|\begin{array}{cc}
a & c \\
a^{\prime} & c^{\prime}
\end{array}\right|>0 \text {. }
$$

In the second case,

$$
\left(\left|\begin{array}{cc}
b & a \\
b^{\prime} & a^{\prime}
\end{array}\right| \cdot\left|\begin{array}{cc}
b & c \\
b^{\prime} & c^{\prime}
\end{array}\right|\right) \cdot\left(\left|\begin{array}{cc}
c & b \\
c^{\prime} & b^{\prime}
\end{array}\right| \cdot\left|\begin{array}{cc}
c & a \\
c^{\prime} & a^{\prime}
\end{array}\right|\right)=\left(\left|\begin{array}{cc}
a & b \\
a^{\prime} & b^{\prime}
\end{array}\right| \cdot\left|\begin{array}{cc}
a & c \\
a^{\prime} & c^{\prime}
\end{array}\right|\right) \cdot\left(\left|\begin{array}{cc}
b & c \\
b^{\prime} & c^{\prime}
\end{array}\right| \cdot\left|\begin{array}{cc}
c & b \\
c^{\prime} & b^{\prime}
\end{array}\right|\right) \leq 0 .
$$

Thus, one of the three products is non-positive

$$
\left|\begin{array}{cc}
a & b \\
a^{\prime} & b^{\prime}
\end{array}\right| \cdot\left|\begin{array}{cc}
a & c \\
a^{\prime} & c^{\prime}
\end{array}\right|, \quad\left|\begin{array}{cc}
b & a \\
b^{\prime} & a^{\prime}
\end{array}\right| \cdot\left|\begin{array}{cc}
b & c \\
b^{\prime} & c^{\prime}
\end{array}\right|, \quad\left|\begin{array}{cc}
c & b \\
c^{\prime} & b^{\prime}
\end{array}\right| \cdot\left|\begin{array}{cc}
c & a \\
c^{\prime} & a^{\prime}
\end{array}\right| .
$$

Proof of Proposition 7 . Interchanging the last two coordinates if necessary, we may assume from (1.5) that $a b^{\prime}-a^{\prime} b \leq 0 \leq a c^{\prime}-a^{\prime} c$, and so

$$
\frac{b^{\prime}}{a^{\prime}} \leq \frac{b}{a} \quad \text { and } \quad \frac{c}{a} \leq \frac{c^{\prime}}{a^{\prime}}
$$

Let $B_{0}$ be the degenerate box with minimum and maximum points

$$
\left(1, \frac{b^{\prime}}{a^{\prime}}, \frac{c}{a}\right) \quad \text { and } \quad\left(1, \frac{b}{a}, \frac{c^{\prime}}{a^{\prime}}\right) \text {. }
$$

The set of lines through the origin that meet $B_{0}$ are exactly those whose whose direction vectors satisfy (1.6). In particular, $B_{0}$ meets $\ell$ and $\ell^{\prime}$. The origin is a (degenerate) box meeting $\ell$ and $\ell^{\prime}$, so their frame-convex hull consists of lines through the origin that meet $B_{0}$, as well as every other box meeting both $\ell$ and $\ell^{\prime}$.

The ascending lines through the origin that meet a given box $B$ form a (double) convex cone with apex the origin. We show that if $\ell$ and $\ell^{\prime}$ meet $B$, then this cone contains the cone over $B_{0}$, which will complete the proof. Scaling coordinates and reflecting $B$ in the origin if necessary, we may assume that $B$ contains the points

$$
\left(s, \frac{s b}{a}, \frac{s c}{a}\right) \in \ell \quad \text { and } \quad\left(1, \frac{b^{\prime}}{a^{\prime}}, \frac{c^{\prime}}{a^{\prime}}\right) \in \ell^{\prime},
$$


for some $s>0$.

Interchanging $\ell$ and $\ell^{\prime}$ if necessary, we may assume that $s \geq 1$. Then the point $\left(s, \frac{s b^{\prime}}{a^{\prime}}, \frac{s c}{a}\right)$ lies on the edge of $B$ beween its vertices $\left(s, \frac{b^{\prime}}{a^{\prime}}, \frac{s c}{a}\right)$ and $\left(s, \frac{s b}{a}, \frac{s c}{a}\right)$. Thus the line $m$ through the first point of (1.7) meets $B$. In a similar fashion, the line $m^{\prime}$ through the second point of (1.7) also meets $B$. These four lines $\ell, \ell^{\prime}, m$, and $m^{\prime}$ generate the cone over $B_{0}$ with apex the origin, which completes the proof.

Thus the frame-convex hull of two general ascending lines that meet in a point $p$ fills out a 2-dimensional quadrilateral cone with vertex $p$. This degenerates to a 1-dimensional cone if the affine span of the lines contains a line through $p$ that is parallel to a coordinate axis. In contrast, the Cremona-convex hull (a subset of the frame-convex hull) is always 1-dimensional and forms part of the ruling of a quadratic cone with vertex $p$, except in these degenerate cases, when it coincides with the frame-convex hull. We illustrate this when the lines have direction vectors $(1,2,3)$ and $(2,3,1)$.

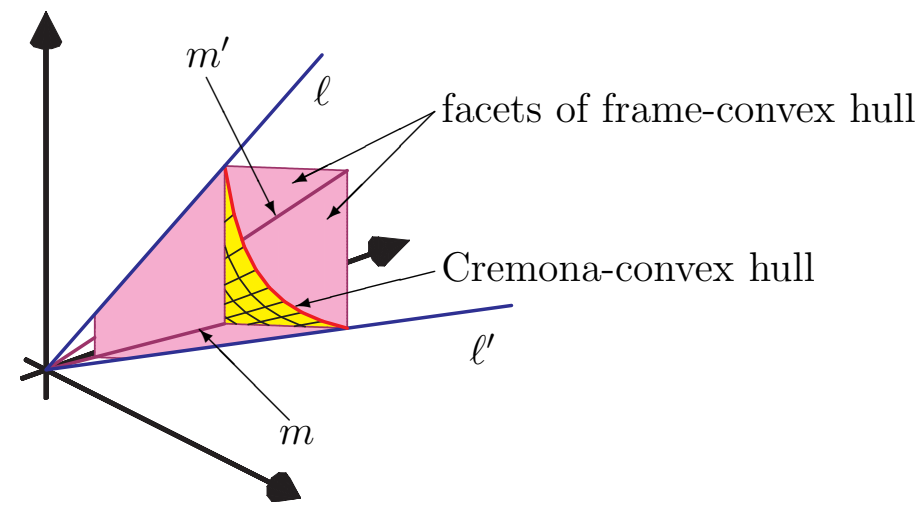

\section{The Cremona transformation for the Grassmannian of Lines}

We describe some of the beautiful geometry behind the Cremona transformation, which is induced by the Cremona coordinates (1.1). This allows us to describe the Cremona-affine hull of up to $d$ ascending lines in $\mathbb{R}^{d}$. A good reference for the vivid classical geometry that we use is Harris's book [9].

2.1. Geometry of the Cremona transformation. The transformation between rectilinear and Cremona coordinates is better understood in terms of homogeneous Plücker coordinates, which are defined by

$$
p_{0 i}=v_{i} \quad \text { and } \quad p_{i j}=x_{i} v_{j}-x_{j} v_{i} \quad \text { for } \quad 1 \leq i<j \leq d .
$$

Let $\mathbb{P}$ be Plücker space, which has $\left(\begin{array}{c}d+1 \\ 2\end{array}\right)$ coordinates. The Cremona transformation

$$
C: \mathbb{P} \rightarrow \mathbb{P} \text { is given by } q=C(p),
$$

where

$$
q_{0 i}=\frac{1}{p_{0 i}} \quad \text { and } \quad q_{i j}=\frac{p_{i j}}{p_{0 i} p_{0 j}}, \quad \text { for } \quad 1 \leq i<j \leq d
$$


Let $\ell$ be a line whose direction vector has no vanishing component. If $(x, v)$ are rectilinear coordinates of $\ell,(y, w)$ are Cremona coordinates of $\ell$ as in (1.1), and $q$ are its Cremona coordinates as in (2.2), then

$$
q_{0 i}=w_{i} \quad \text { and } \quad q_{i j}=y_{i}-y_{j}, \quad \text { for } \quad 1 \leq i<j \leq d,
$$

which does not depend upon the choice of $y$. This Cremona transformation is an isomorphism outside the coordinate hyperplanes $p_{0 i}=0$ for $i=1, \ldots, d$. It is also an involution, since applying it twice on points where it is an isomorphism gives the identity.

Remark 9. While this agrees with the notion of Cremona coordinates of (1.1) if no Plücker coordinate $p_{0 i}$ vanishes, these two notions differ for lines where some of these coordinates vanish. (Recall that $\left(p_{01}, \ldots, p_{0 d}\right)$ is the direction vector of a line with Plücker coordinates $p_{i j}$ ). That is because the Cremona tranformation is continuous where it is defined (which is described in Theorem 10), whereas the assignment of Cremona coordinates (1.1) is not continuous (but defined everywhere).

The Grassmannian of lines $G(1, d)$ in Plücker space is defined by the Plücker relations

$$
\begin{aligned}
p_{0 i} p_{j k}-p_{0 j} p_{i k}+p_{0 k} p_{i j}=0 & \text { for } \quad 1 \leq i<j<k \leq d, \quad \text { and } \\
p_{i j} p_{k l}-p_{i k} p_{j l}+p_{i l} p_{j k}=0 & \text { for } \quad 1 \leq i<j<k<l \leq d .
\end{aligned}
$$

Let $1 \leq i<j<k \leq d$. Dividing the Plücker relation

$$
p_{0 i} p_{j k}-p_{0 j} p_{i k}+p_{0 k} p_{i j}=0
$$

by $p_{0 i} p_{0 j} p_{0 k}$ reduces it to the linear relation

$$
q_{j k}-q_{i k}+q_{i j}=0 .
$$

Let $1 \leq i<j<k<l \leq d$. Dividing the Plücker relation

$$
p_{i j} p_{k l}-p_{i k} p_{j l}+p_{i l} p_{j k}=0
$$

by $p_{0 i} p_{0 j} p_{0 k} p_{0 l}$ reduces it to

$$
\begin{aligned}
& q_{i j} q_{k l}-q_{i k} q_{j l}+q_{i l} q_{j k}= \\
& q_{k l}\left(q_{i j}-q_{i k}+q_{j k}\right)+q_{i k}\left(q_{j k}-q_{j l}+q_{k l}\right)+q_{j k}\left(q_{i k}-q_{i l}+q_{k l}\right)=0 .
\end{aligned}
$$

Therefore the Cremona transformation maps $G(1, d)$ into the linear subspace $L G(d)$ :

$$
q_{j k}-q_{i k}+q_{i j}=0, \quad \text { for } \quad 1 \leq i<j<k \leq d .
$$

We may use these linear relations to express any $q_{j k}$ with $1<j$ in terms of the coordinates $q_{1 i}$. Since no equation defining $L G(d)$ involves any $q_{0 i}$, these relations define a linear subspace of dimension $2 d-2$, which equals the dimension of the Grassmannian. Since the Cremona transformation is an isomorphism outside the coordinate hyperplanes $p_{0 i}=0$, we see that $G(1, d)$ is mapped birationally onto the linear space $L G(d)$.

Theorem 10. The birational Cremona transformation $C$ on Plücker space is an involution that maps $G(1, d)$ to $L G(d)$ birationally. The indeterminancy locus of $C$ has codimension 3 and consists of the linear subspaces

$$
\begin{aligned}
L_{i j k}: & p_{0 i}=p_{0 j}=p_{0 k}=0 \quad \text { for } 1 \leq i<j<k \leq d, \quad \text { and } \\
L_{i j}: & p_{i j}=p_{0 i}=p_{0 j}=0 \text { for } 1 \leq i<j \leq d .
\end{aligned}
$$


The indeterminacy locus of the Cremona transformation restricted to $G(1, d)$ has $\left(\begin{array}{c}d \\ 2\end{array}\right)+1$ components, all of codimension 2. One component is the set of lines at infinity, while the other components consist of the sets of lines whose direction vectors have at least two coordinates equal to 0.

The indeterminancy locus of the Cremona transformation restricted to $L G(d)$ has codimension 3 and consists of the intersections of the subspaces $L_{i j k}$ and $L_{i j}$ with $L G(d)$.

This indeterminacy locus in $L G(d)$ is the image under $C$ of lines whose direction vectors have one coordinate equal to 0 .

The set of lines in $\mathbb{P}^{d}$ that meet a linear subspace having codimension $a+1$ is a special Schubert cycle. This irreducible subvariety has codimension $a$ in $G(1, d)$. More generally, given subspaces $L \subsetneq M$ of $\mathbb{P}^{d}$ where $L$ has codimension $a+1$ and $M$ has codimension $b$, then the set of lines lying in $M$ that meet $L$ is a Schubert cycle. This has codimension $a+b$ in $G(1, d)$ and is special when $b=0$.

Proof. We have already noted that Cremona transformation is an involution that restricts to a birational map from $G(1, d)$ to $L G(d)$. If we multiply the coordinates of the Cremona transformation (2.2) by $p_{01} p_{02} \cdots p_{0 d}$, we see that it is also given by

$$
\begin{array}{ll}
q_{0 i}=p_{01} \cdots \widehat{p_{0 i}} \cdots p_{0 d} & \text { for } \quad 1 \leq i \leq d, \quad \text { and } \\
q_{i j}=p_{i j} p_{01} \cdots \widehat{p_{0 i}} \cdots \widehat{p_{0 j}} \cdots p_{0 d} & \text { for } \quad 1 \leq i<j \leq d,
\end{array}
$$

and is therefore undefined if either

(i) any three coordinates $p_{0 i}, p_{0 j}, p_{0 k}$ vanish, or

(ii) any two coordinates $p_{0 i}, p_{0 j}$ vanish, and we have $p_{i j}=0$.

But (i) defines the subspace $L_{i j k}$ and (ii) defines the subspace $L_{j k}$.

The restriction of these linear spaces to $G(1, d)$ is conveniently described using special Schubert cycles. The hyperplane section $p_{0 i}=0$ is the set of lines that meet the codimension 2 linear subspace $z_{0}=z_{i}=0$, which is a hyperplane at infinity. Similarly, the hyperplane section $p_{i j}=0$ is the set of lines that meet the codimension 2 linear subspace defined by $z_{i}=z_{j}=0$. Therefore $L_{i j k} \cap G(1, d)$ has two components: the set of lines at infinity, and the set of lines that meet the codimension 4 subspace $z_{0}=z_{i}=z_{j}=z_{k}=0$. The first of these sets is a special Schubert cycle of codimension 2, while the other is a special Schubert cycle of codimension 3 in $G(1, d)$. Similarly, $L_{i j} \cap G(1, d)$ is the set of lines that meet the codimension 3 linear subspace $z_{0}=z_{i}=z_{j}=0$. This is a special Schubert cycle of codimension 2 that contains the second component of the intersection $L_{i j k} \cap G(1, d)$.

Finally, the linear forms defining either $L_{i j k}$ or $L_{i j}$ are linearly independent of those defining $L G(d)$, therefore they define codimension 3 linear subspaces of $L G(d)$ as well. This indeterminacy locus is the image under $C$ of the coordinate hyperplane sections $p_{0 i}=0$ of $G(1, d)$. The direction vector of such lines has some coordinate equal to 0 .

The restriction of the Cremona transformation to coordinate hyperplanes $p_{0 i}=0$, outside the indeterminacy locus, is a contraction.

Proposition 11. The coordinate hyperplane $p_{0 i}=0$ meets the Grassmannian $G(1, d)$ in the Schubert cycle of lines that meet the codimension 2 plane $z_{0}=z_{i}=0$ at infinity. It is contracted by $C$ to a line in $L G(d)$. 
Proof. The Plücker coordinates of a line that meets the codimension 2 plane $z_{0}=z_{i}=0$ have $p_{0 i}=0$, so its only nonzero Cremona coordinates are $q_{0 i}$ and $q_{1 i}=\cdots=q_{i d}$.

2.2. Cremona-affine span of lines. Recall that if $f: X \rightarrow Y$ is a rational map with $Z \subset X$ an irreducible subvariety that is not contained in the indeterminacy locus $W \subset X$ of the map $f$, then the (proper) image of $Z$ is the closure $\overline{\varphi(Z \backslash W)}$ in $Y$ of its set-theoretic image. Given a set $\Gamma$ of lines, their Cremona coordinates span a linear subspace $L(\Gamma)$ in $L G(d)$ whose image $G(\Gamma)$ in $G(1, d)$ under the Cremona transformation is the Cremonaaffine span of the original lines. We use the Cremona map of Section 2.1 to obtain a precise description of such Cremona-affine spans.

Since the indeterminacy locus of the Cremona transformation has codimension 3 when restricted to $L G(d)$ we are able to describe the subvarieties of the Grassmannian that correspond to general linear spaces of lines of dimension up to 3 in Cremona coordinates. A 2- or 3-dimensional subvariety $X$ of the Grassmannian has a bidegree $\left(d_{1}, d_{2}\right)$ given by its intersection with general Schubert cycles of codimension 2 and 3 respectively. In codimension $2, d_{1}$ counts the number of lines in $X$ that meet a general codimension 3 linear space, while $d_{2}$ counts the number of lines that lie in a general hyperplane. In codimension $3, d_{1}$ counts the number of lines in $X$ that meet a general codimension 4 linear space, while $d_{2}$ counts the number of lines that lie in a hyperplane and meet a codimension 2 linear subspace of this hyperplane.

A linear subspace in $L G(d)$ is in general position if it meets the indeterminacy locus of $C$ properly, that is, in a set of codimension 3. The Cremona coordinates $q_{0 i}$ of a line are the inverses of the components of its direction vector. We call the vector $\left(q_{01}, \ldots, q_{0 d}\right)$ the Cremona direction of the line. A set of $n \leq d$ lines are independent if their Cremona directions are affinely independent. If their affine span is in addition in general position in $L G(d)$, then they are in general position.

Note that a set of independent lines have affinely independent images in $L G(d)$, while the converse is not true. The converse fails for instance when two lines have the same direction.

Theorem 12. Let $\Gamma$ be a set of $n$ independent lines in general position in $\mathbb{R}^{d}$. Then their Cremona-affine span $G(\Gamma)$ is a rational $(d-1)$-fold in $G(1, d)$.

(1) If $n=2$, then $G(\Gamma)$ is a rational normal curve of degree $d-1$.

(2) If $n=3$, then $G(\Gamma)$ is a Veronese surface of bidegree $\left(\left(\begin{array}{c}d-1 \\ 2\end{array}\right),\left(\begin{array}{l}d \\ 2\end{array}\right)\right)$ and degree $(d-1)^{2}$.

(3) If $n=4$, then $G(\Gamma)$ is a rational threefold of bidegree $\left(\left(\begin{array}{c}d-1 \\ 3\end{array}\right), 2\left(\begin{array}{l}d \\ 3\end{array}\right)\right)$ and degree $(d-1)^{3}-\left(\begin{array}{l}d \\ 3\end{array}\right)-\left(\begin{array}{l}d \\ 2\end{array}\right)$.

Proof. Clearly these varieties are rational. For the numerical results, we compute the bidegree and degree of $G(\Gamma)$. The Cremona transformation as expressed in (2.4) is defined by polynomials of degree $d-1$. The indeterminacy locus has codimension 3 , so for $k=2$ and $k=3$ the degree of $G(\Gamma)$ is $(d-1)^{k-1}$. For $k=3$ the indeterminacy locus consists of the points of intersection with $L_{i j k}$ and $L_{i j}$, which add up to $\left(\begin{array}{l}d \\ 3\end{array}\right)+\left(\begin{array}{l}d \\ 2\end{array}\right)$ points, from which the degree of $G(\Gamma)$ follows. The bidegree is determined by the degree of $G(\Gamma)$ and the degree of the corresponding union of lines in $\mathbb{R}^{d}$. The latter can be computed by considering a codimension $k-1$ subspace of the hyperplane at infinity, and counting how many lines in the family meet this subspace. This is determined by the direction vector 
of the line, which consists of its Plücker coordinates $p_{0 i}(2.1)$. On these coordinates the Cremona transformation is the ordinary Cremona transformation defined by inverting all the coordinates. The degree of the restriction of an ordinary Cremona transformation on $\mathbb{P}^{m}$ to a general $n$-dimensional linear subspace is $\left(\begin{array}{c}m \\ n\end{array}\right)$. Therefore the degree of the image of a $(k-1)$-dimensional linear subspace at infinity is $\left(\begin{array}{l}d-1 \\ k-1\end{array}\right)$.

We describe the Cremona-affine hull of a finite set of lines whose direction vectors have nonzero coordinates, as a subset of $\mathbb{R}^{d}$. Consider the homogeneous Plücker coordinates $p_{i j}$ for an ascending line $\ell$. We may choose two points on the line with coordinates

$$
\left(\begin{array}{ccccc}
1 & 0 & -p_{12} & \ldots & -p_{1 d} \\
0 & 1 & p_{02} & \ldots & p_{0 d}
\end{array}\right)
$$

We have scaled the Plücker coordinates so that $p_{01}=1$. This is possible by the assumption that the coordinates $p_{0 i}$ are nonzero. The linear forms vanishing on $\ell$ have a basis

$$
\begin{aligned}
b_{i}(\ell) & =\frac{p_{1 i}}{p_{0 i}} z_{0}-z_{1}+\frac{1}{p_{0 i}} z_{i} \\
& =q_{1 i} z_{0}-z_{1}+q_{0 i} z_{i}, \quad \text { for } \quad i=2, \ldots, d .
\end{aligned}
$$

These are Santaló's equations (1.3), expressed in the Cremona coordinates (2.3).

Given a set of $n$ lines $\Gamma=\left\{\ell_{1}, \ldots, \ell_{n}\right\}$, let $M(\Gamma)$ be the $n \times(d-1)$-matrix of linear forms with entries $b_{i}\left(\ell_{j}\right)$. As in Remark [5, a linear combination of the rows of $M(\Gamma)$ gives a vector of linear forms whose coefficients are the same linear combination of the Cremona coordinates of the $\ell_{i}$. This identifies the row space of $M(\Gamma)$ with the affine span of the Cremona coordinates of the lines $\ell_{i}$. The matrix $M(\Gamma)$ provides a determinantal description of the variety of lines $S(\Gamma) \subset \mathbb{R}^{d}$ that belong to the linear span of $\Gamma$ in Cremona coordinates.

Theorem 13. Let $\Gamma$ be a set of $n$ independent lines in $\mathbb{R}^{d}$ whose direction vectors have only nonzero coordinates. Let $L(\Gamma) \subset L G(d)$ be the linear span of their Cremona coordinates, $G(\Gamma)$ their Cremona-affine span, and $S(\Gamma) \subset \mathbb{R}^{d}$ the union of the lines in $G(\Gamma)$.

(1) The set of lines in the family $G(\Gamma)$ that pass through a point in $\mathbb{R}^{d}$ is linear in $L(\Gamma)$.

(2) When $n \leq d-1, S(\Gamma)$ is a $n$-fold scroll of degree $\left(\begin{array}{l}d-1 \\ n-1\end{array}\right)$ defined by the maximal minors of $M(\Gamma)$.

(3) The matrix $M(\Gamma)$ has rank at most $r$ precisely at the points in $\mathbb{R}^{d}$ that are contained in a $(n-r-1)$-dimensional set of lines in the family.

(4) If $n \leq d$, there is at most one line in the family in each direction with only nonzero coordinates.

(5) For $n=d$ the set of points in $\mathbb{R}^{d}$ that lie on more than one line in the family form a codimension 2 subvariety $Z(\Gamma)$ in $\mathbb{R}^{d}$ of degree $\left(\begin{array}{l}d \\ 2\end{array}\right)$. In particular, $G(\Gamma)$ is the set of $(d-1)$-secant lines to $Z(\Gamma)$.

Part (2) for $n=2$ and $d=3$ and Part (5) for $d=3$ are illustrated in Figure 1. Notice that the degree of the union of lines in $\mathbb{R}^{d}$ of the family $G(\Gamma)$ coincides with the number of lines in the family that meet a general codimension $n$ linear subspace.

Proof. The set of lines in the family that pass though a point are cut out by linear combinations of rows in $M(\Gamma)$ that vanish when evaluated at that point, so this set is a linear subspace of $L(\Gamma)$. If the rank of the matrix $M(\Gamma)$ at $p$ is $r<n$, then the dimension of this 
set of lines is $n-r-1$. The union of all lines in the family is precisely the $n$-dimensional subvariety of $\mathbb{R}^{d}$ where the matrix has rank at most $n-1$. Its degree is given by the ThomPorteus formula [4, p. 254]. When $n=d$, the matrix $M(\Gamma)$ has dimensions $n \times(n-1)$. It therefore has rank at most $n-2$ on a $(d-2)$-dimensional subvariety $Z(\Gamma)$ of degree $\left(\begin{array}{l}d \\ 2\end{array}\right)$ in $\mathbb{R}^{d}$, defined by the maximal minors of $M(\Gamma)$. For each line in the family, this intersection with $Z(\Gamma)$ is defined by a unique $(d-1)$-dimensional minor, so the line is $(d-1)$-secant to $Z(\Gamma)$. On the other hand, let $L$ be a $(d-1)$-secant line to $Z(\Gamma)$. Then the subspace of maximal minors of $M(\Gamma)$ that vanish on $\ell$ has codimension 1 . But the codimension 1 subspaces of rows of the matrix correspond naturally to this space of minors, so the codimension 1 subspaces corresponding to minors that vanish on $\ell$ have a unique row in common. This row must define the line $\ell$.

Let $n<d$. Note that the matrix $M(\Gamma)$ has rank 1 at infinity along the $i$-th coordinate axis (where $z_{j}=0$ for $j \neq i$ ). Therefore there is a $(n-1) \times(d-1)$-submatrix $M_{i}(\Gamma)$ of $M(\Gamma)$ that has rank 0 at infinity along the $i$-th coordinate axis.

Corollary 14. Let $\Gamma$ be a set of $n$ independent lines whose direction vectors have only nonzero coordinates in $\mathbb{R}^{d}$, where $1<n<d$. The lines in the family $G(\Gamma)$ that are parallel to the $i$-th coordinate axis form a $(n-2)$-dimensional family $G_{i}(\Gamma) \subset G(1, d)$ which is the preimage under $C$ of the codimension 1 linear subspace $L_{i}(\Gamma)=\left\{q_{0 i}=0\right\} \cap L(\Gamma)$. The corresponding subvariety $S_{i}(\Gamma) \subset S(\Gamma)$ is an $(n-1)$-dimensional cylinder over an $(n-2)$ dimensional subvariety of the coordinate hyperplane $\left\{z_{i}=0\right\}$ defined by the maximal minors of the $(n-1) \times(d-1)$-matrix $M_{i}(\Gamma)$. In particular, $S_{i}(\Gamma)$ is a cylinder over a rational determinantal variety of degree $\left(\begin{array}{c}d-1 \\ n-2\end{array}\right)$.

We characterize some sets $\Gamma$ of independent lines and some general linear subspaces $L(\Gamma)$.

Proposition 15. Two lines whose direction vectors have only nonzero coordinates are independent if their direction vectors are distinct. Three lines whose direction vectors have only nonzero coordinates are independent if and only if there is no surface of minimal degree $d-1$ that contains all three and passes through the coordinate directions at infinity.

Proof. The first statement is the definition of independent. For the second statement, notice that the construction of the matrix $M(\Gamma)$ defines the unique scroll of minimal degree that contains two lines whose direction vectors have only nonzero coordinates and that passes through the coordinate directions at infinity.

Given two lines in $\mathbb{R}^{d}$ whose direction vectors have only nonzero coordinates, then the scroll of degree $d-1$ that is defined by their Cremona-affine span contains one line parallel to each coordinate axis. This line has a direct synthetic description: Consider two lines $\ell_{1}, \ell_{2}$, and the coordinate simplex $\Delta$ at infinity. For each codimension 2 subsimplex $\Delta^{\prime} \subset \Delta$, consider the codimension 2 subspace $P_{\Delta^{\prime}}$ through $\Delta^{\prime}$ that meets both lines $\ell_{1}, \ell_{2}$. There is a unique line through each vertex of $\Delta$ that meets all the subspaces $P_{\Delta^{\prime}}$. This is the line of the scroll parallel to the chosen coordinate axis.

Together with the original lines $\ell_{1}, \ell_{2}$ we count $d+2$ lines that meet $\left(\begin{array}{l}d \\ 2\end{array}\right)$ codimension 2 linear spaces $P_{\Delta^{\prime}}$. The set of lines that meet a codimension 2 linear space form a hyperplane section of $G(1, d)$. In general, the hyperplanes defined by the different $P_{\Delta^{\prime}}$ are linearly 
independent, and therefore define a $\mathbb{P}^{d-1}$ inside Plücker space. The $d+2$ lines are represented by $d+2$ points in this space. By Castelnuovo's lemma [7, p. 530], there is a unique rational normal curve through these $d+2$ points. This curve defines the scroll $S\left(\ell_{1}, \ell_{2}\right)$ of Theorem 13 .

It is straightforward to verify the following properties of the scroll $S\left(\ell_{1}, \ell_{2}\right)$.

Proposition 16. Let $\ell_{1}$ and $\ell_{2}$ be two lines whose direction vectors have only nonzero coordinates. If they are disjoint, then their span $S\left(\ell_{1}, \ell_{2}\right)$ is a smooth scroll. If they meet at a finite point, then their span is a cone over a rational normal curve.

Example [6] illustrated the case of Proposition [16] in $\mathbb{R}^{3}$ where the two lines have nonvanishing coordinate directions and they meet.

Proposition 17. Let $\ell_{1}, \ell_{2}$, and $\ell$ be three lines in $\mathbb{R}^{d}$ whose direction vectors have only nonzero coordinates. Assume that the two lines $\ell_{1}$ and $\ell_{2}$ are independent and have direction vectors whose affine span does not meet any codimension 2 coordinate plane. Then the following are equivalent:

(1) $\ell$ meets every codimension 2 plane parallel to two coordinate hyperplanes that also meet $\ell_{1}$ and $\ell_{2}$.

(2) The projections of the three lines into any coordinate plane have a common point.

(3) $\ell$ is a ruling in the unique scroll of minimal degree that contains $\ell_{1}$ and $\ell_{2}$ and passes through the coordinate directions at infinity.

Proof. Since the span of the direction vectors of $\ell_{1}$ and $\ell_{2}$ does not intersect any codimension 2 coordinate plane at infinity, the projection of the two lines to any coordinate plane meet in a unique point. The preimage of this point is a codimension 2 plane parallel to two coordinate hyperplanes that meet both lines, and any such codimension 2 plane arises this way. Therefore (1) and (2) are equivalent.

Let $H_{i j}$ be the unique codimension 2 plane that intersects both $\ell_{1}$ and $\ell_{2}$ and is parallel to all coordinate axes except the $i$ - and the $j$-axis.

For (3), note that by Propositions [15] and [16 the scroll $S\left(\ell_{1}, \ell_{2}\right)$ is smooth and uniquely determined by the two lines and the coordinate directions at infinity. The intersection $C_{i j}=H_{i j} \cap S\left(\ell_{1}, \ell_{2}\right)$ contains a point at infinity on $d-2$ coordinate axes in addition to one point on each of the lines $\ell_{1}$ and $\ell_{2}$. Altogether we have counted $d$ points, while the scroll has degree only $d-1$. Therefore the intersection $C_{i j}$ contains a curve which furthermore must intersect every line in the ruling of $S\left(\ell_{1}, \ell_{2}\right)$ defined by $\ell_{1}$ and $\ell_{2}$. In particular, $\ell$ must intersect $H_{i j}$ if it belongs to the scroll $S\left(\ell_{1}, \ell_{2}\right)$.

On the other hand, the Schubert cycle of lines meeting $H_{i j}$ forms a hyperplane section $\Lambda_{i j} \cap G(1, d)$ of $G(1, d)$ in $\mathbb{P}$. The image of $\Lambda_{i j}$ under the Cremona transformation is a hyperplane $\Lambda_{i j}^{\prime}$ that meets $L G(d)$ in a hyperplane $P_{i j}$ of $L(G)$. If $\ell$ meets each $H_{i j}$, its Cremona coordinates must lie in every $P_{i j}$. Parallel to each coordinate axis there is exactly one line that meets each $H_{i j}$, so the intersection of the hyperplanes $P_{i j}$ is exactly the line spanned by the Cremona coordinates of $\ell_{1}$ and $\ell_{2}$. Therefore the Cremona coordinates of $\ell$ belong to this line, and $\ell$ belongs to $S\left(\ell_{1}, \ell_{2}\right)$.

We will use this to show that the frame-convex hull of two general ascending lines equals their Cremona-convex hull. 
First note that these two notions of convexity are preserved by orthogonal projections along coordinate directions. Indeed, suppose that $\ell$ is a (necessarily) ascending line lying in the frame-convex hull of two ascending lines $\ell_{1}$ and $\ell_{2}$ and let $\pi$ be an orthogonal projection to a coordinate $d^{\prime}$-plane. Then $\pi(\ell)$ lies in the frame-convex hull of $\pi\left(\ell_{1}\right)$ and $\pi\left(\ell_{2}\right)$. The same is true for Cremona convexity.

Suppose that $\ell_{1}$ and $\ell_{2}$ are ascending lines with different direction vectors. Then their Cremona-affine hull $G\left(\ell_{1}, \ell_{2}\right)$ consists of a rational curve of lines with different directions, and the lines $\ell_{1}$ and $\ell_{2}$ divide this curve (topologically a circle) into two intervals, one of which consists of ascending lines.

Corollary 18. Suppose that $\ell_{1}$ and $\ell_{2}$ are ascending lines with different direction vectors. Then their Cremona-convex hull is the interval they define on $G\left(\ell_{1}, \ell_{2}\right)$ consisting of ascending lines. If $d=2$ or if the two lines are disjoint and have direction vectors whose affine span does not meet any codimension 2 coordinate plane, then this is also their frame-convex hull.

Proof. The first statement is straightforward: Since $\ell_{1}$ and $\ell_{2}$ are ascending, every line in their Cremona-convex hull is also ascending. But this Cremona-convex hull is one of the intervals they define on $G\left(\ell_{1}, \ell_{2}\right)$.

Suppose now that either $\ell_{1}$ and $\ell_{2}$ are skew and have direction vectors whose affine span does not meet any codimension 2 coordinate plane or else $d=2$. By assumption, their images under any orthogonal projection $\pi$ to a coordinate 2-plane meet in a unique point $p$, and the inverse image $\pi^{-1}(p)$ is a codimension 2 plane $H$ that is parallel to the codimension 2 coordinate plane $\pi^{-1}(0)$. Let $B(\subset H)$ be the smallest box containing the points $\ell_{1} \cap H$ and $\ell_{2} \cap H$ (necessarily as vertices). Thus the frame-convex hull of $\ell_{1}$ and $\ell_{2}$ is a subset of the set of lines meeting each such coordinate-parallel codimension 2 plane $H$, but this larger set is the Cremona-affine hull of $\ell_{1}$ and $\ell_{2}$, by Proposition 17.

Choose a different projection $\pi^{\prime}$ to a coordinate 2-plane in which the box $B$ projects to an interval of positive length. The endpoints of this interval are necessarily the projections of the points $\ell_{1} \cap H$ and $\ell_{2} \cap H$, and we have $\pi^{\prime}\left(\ell_{1}\right) \neq \pi^{\prime}\left(\ell_{2}\right)$. Since $\pi^{\prime}$ is $1-1$ on lines with no vanishing coordinate directions, in particular on ascending lines, it maps lines in the frame-convex hull of $\ell_{1}$ and $\ell_{2}$ to lines in the frame-convex hull of $\pi^{\prime}\left(\ell_{1}\right)$ and $\pi^{\prime}\left(\ell_{2}\right)$. This is the interval of lines in that coordinate plane through $p:=\pi^{\prime}\left(\ell_{1}\right) \cap \pi^{\prime}\left(\ell_{2}\right)$ consisting of ascending lines, and so is also the image of the Cremona-convex hull of $\ell_{1}$ and $\ell_{2}$. Since $\pi^{\prime}$ is 1-1 on ascending lines, this shows that the frame-convex hull of $\ell_{1}$ and $\ell_{2}$ equals their Cremona-convex hull.

\section{Convexity structures For AfFine subspaces}

We discuss convexity structures for general affine subspaces of $\mathbb{R}^{d}$. We first describe Santaló's Helly-type theorem for hyperplanes, and then give a Helly-type result for codimension 2 linear subspaces via their Cremona coordinates. Next, we discuss frame convexity for lines with different sign patterns, and finally show that frame convexity for $k$-flats in $\mathbb{R}^{d}$ with $1<k<d-1$ has a completely different character than for points, lines, or hyperplanes.

Santaló also proved the following Helly-type theorem for hyperplane transversals. 
Theorem 19. If every $2^{d-1}(d+1)$ (or fewer) members of a collection of boxes in $\mathbb{R}^{d}$ admit a hyperplane transversal, then there is a hyperplane transversal to all the boxes.

As with Theorem 1, this will be implied by a local version. A hyperplane $H$ is ascending if it has equation

$$
z \cdot v=\sum_{i=1}^{d} z_{i} v_{i}=c,
$$

where the perpendicular vector $v=\left(v_{1}, \ldots, v_{d}\right)$ is ascending. An ascending hyperplane meets a box with minimum point $a$ and maximum point $b$ if and only if

$$
a \cdot v \leq c \leq b \cdot v \text {. }
$$

Thus the set of ascending hyperplanes meeting this box is convex, in the coordinates given by the coefficients of their defining equations. Theorem 19] follows immediately from Helly's theorem, in the same way as Theorem 11.

Remark 20. The convexity for both ascending lines and ascending hyperplanes in $\mathbb{R}^{d}$ came from natural coordinates that identified them as convex subsets of $\mathbb{R}^{2 d-2}$ and $\mathbb{R}^{d}$, respectively. For hyperplanes, this is quite natural, as the set of hyperplanes in $\mathbb{P}^{d}$ is just the dual $\mathbb{P}^{d}$. For lines, the Cremona transformation linearizes the Grassmannian of lines, and our notion of Cremona convexity is pulled back from the affine structure on this linearized Grassmannian. For $1<k<d-2$, there is no linearizing Cremona transformation for the Grassmannian of $k$-flats in $\mathbb{P}^{d}$, which partially explains our inability to extend the results for lines and hyperplanes to $k$-flats for arbitrary $k$.

The other ingredient in these results is that the natural convex structure from the linearizations has the nice geometric interpretation of frame convexity. Since the set of $(d-2)$ flats is isomorphic to the space of lines, there is a linearizing Cremona transformation and a notion of convexity for $(d-2)$-flats. It does not, however have as nice a geometric interpretation as frame convexity. We describe it by dualizing the notion of frame convexity.

The dual of a $k$-flat in $\mathbb{R}^{d}$ is a $(d-1-k)$-flat in $\mathbb{R P}^{d}$ that does not meet the origin. We restrict ourselves to flats in $\mathbb{R P}^{d}$ that do not meet the origin. Every hyperplane (at finite distance) not containing the origin has a unique equation of the form

$$
a \cdot z=\sum_{i} a_{i} z_{i}=1
$$

where $z_{1}, \ldots, z_{d}$ are the coordinates of $\mathbb{R}^{d}$. The coefficients $a=\left(a_{1}, \ldots, a_{d}\right)$ give coordinates for hyperplanes not containing the origin, with $(0, \ldots, 0)$ giving the hyperplane at infinity. The set of such hyperplanes is partially ordered by componentwise comparison of these coordinates. This has a geometric interpretation.

Order the non-zero points on a coordinate axis in $\mathbb{P}^{d}$ so that the positive numbers with their usual order precede the point at infinity, which precedes the negative numbers with their usual order. Given two hyperplanes $A$ and $B$ not containing the origin, we have that $A \leq B$ if and only if for each coordinate axis $\ell, \ell \cap A$ precedes $\ell \cap B$ in this order. The $*-b o x$ between two hyperplanes $A \leq B$ not containing the origin is the set of those hyperplanes $H$ (not containing the origin) that satisfy $A \leq H \leq B$. 
A $(d-2)$-flat $K$ not meeting the origin is ascending if its span with the origin is an ascending hyperplane. That is, $K$ is the set of points $z$ in $\mathbb{R}^{d}$ defined by the equations

$$
z \cdot x=1, \quad \text { and } \quad z \cdot v=0,
$$

where each coordinate of $v$ is non-negative and $v \neq 0$. This pair $(x, v)$ gives rectilinear coordinates for $K$, and we obtain its Cremona coordinates via the transform (1.1).

The hyperplanes containing $K$ that do not contain the origin have the form

$$
H_{t}:\left\{z \in \mathbb{R}^{d} \mid z \cdot(x+t v)=1\right\}, \quad \text { for } t \in \mathbb{R} .
$$

An ascending $(d-2)$-flat $*$-transverse to a $*$-box defined by hyperplanes $A \leq B$ is an ascending 2-plane lying on a hyperplane $H$ in the $*$-box.

Since these definitions are just a translation of those for lines to $(d-2)$-flats via duality, we have the following results.

Theorem 21. The set of Cremona coordinates of ascending $(d-2)$-flats that are $*$-transverse to a given *-box is convex.

Corollary 22. If we have a collection of $*$-boxes in $\mathbb{R}^{d}$ such that every $2 d-1$ of them have $a *$-transversal, then they all do.

Note that $*$-transversality makes sense for any $(d-2)$-flat that does not contain the origin. Here is Santaló's theorem in this context.

Theorem 23. If every $2^{d-1}(2 d-1)$ (or fewer) members of a collection of $*$-boxes in $\mathbb{R}^{d}$ admit $a *$-transversal by a $(d-2)$-flat, then there is a $(d-2)$-flat $*$-transversal to all the *-boxes.

Example 24. While the frame-convex hull of two general ascending lines has dimension 1 , if the lines have different sign pattern, then their frame-convex hull is just the two lines again, as the figure below shows for the lines $\ell_{1}$ and $\ell_{2}$, and the three axis-parallel boxes (actually segments) $B_{1}, B_{2}$, and $B_{3}$.

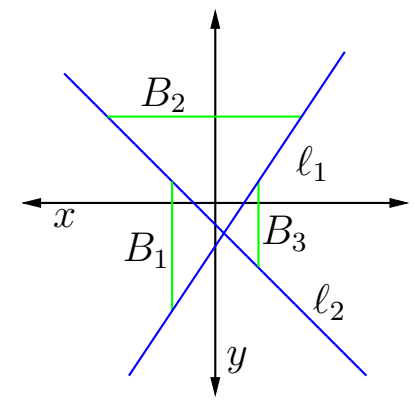

When $k \neq 1, d-1$, frame convexity for $k$-flats in $\mathbb{R}^{d}$ has a completely different character than when $k$ is 1 or $d-1$. For example, the frame-convex hull of two 2-flats in either $\mathbb{R}^{4}$ or $\mathbb{R}^{5}$ is just the two original 2-flats.

Proposition 25. The frame-convex hull of any two 2-flats in general position in either $\mathbb{R}^{4}$ or $\mathbb{R}^{5}$ consists solely of the original 2-flats. 
Proof. We give an algebraic relaxation of the frame-convex hull whose only solutions are the original 2-flats. A $k$-flat in $\mathbb{R}^{d}$ corresponds to a $(k+1)$-dimensional linear subspace of $\mathbb{R}^{d+1}$, which we represent as the row space of a $(k+1) \times(d+1)$ matrix, and consider as a $k$-flat in projective space $\mathbb{R} \mathbb{P}^{d}$. Consider the two 2 -flats in $\mathbb{R P}^{5}$

$$
\left[\begin{array}{rrrrrr}
1 & 1 & 1 & 1 & 1 & 1 \\
1 & -1 & -1 & -1 & 1 & 0 \\
0 & -1 & 1 & 2 & 2 & 0
\end{array}\right] \quad \text { and } \quad\left[\begin{array}{rrrrrr}
-1 & -1 & -1 & -1 & -1 & 1 \\
-1 & 1 & -1 & 1 & -1 & 0 \\
0 & -1 & 2 & 1 & 2 & 0
\end{array}\right] \text {. }
$$

For each coordinate $x_{i}$, there is a line parallel to the $x_{i}$-axis meeting both 2-planes. For each, we give the point in $\mathbb{R P}^{5}$ on the line with $x_{i}=0$.

$$
\begin{aligned}
& x_{1} \text {-axis }:(0,-3,13,21,13,1) \\
& x_{2} \text {-axis }:(3,0,-5,-9,-5,1) \\
& x_{3} \text {-axis }:(-1,1,0,1,-1,0) \\
& x_{4} \text {-axis }:(1,1,-3,0,-3,0) \\
& x_{5} \text {-axis }:(9,1,-15,-23,0,1)
\end{aligned}
$$

Among the boxes meeting our two 2-flats are axis-parallel segments lying along these axisparallel transversals. Thus the set $X$ of 2 -flats meeting these axis-parallel transversals contains the frame-convex hull of our original 2-flats. We consider $X$ to be an algebraic relaxation of the frame-convex hull.

Formulating and solving the equations for a general 2-plane in $\mathbb{P}^{5}$ to meet each of these five lines shows that $X$ consists of our original two 2-planes. This is not unexpected: the space of 2-planes in $\mathbb{P}^{5}$ has dimension 9 , and the set of 2 -planes meeting a line has codimension 2 , so there would be be no solutions to such a geometric problem, if the lines were in general position. Since this algebraic relaxation for a particular choice of two 2-flats has only the two original 2-flats as solutions, this will be the case for any two general 2-flats in $\mathbb{R}^{5}$.

A different procedure shows that the solutions to an algebraic relaxation to the frameconvex hull of two 2-flats in $\mathbb{R}^{4}$ consist of only those 2-flats. If we choose a third 2-flat, then there are four axis-parallel lines meeting the three. Choosing yet another 2-flat gives four more axis-parallel lines, and an algebraic relaxation of the frame-convex hull of the original two 2-flats is the set of 2-flats meeting all 8 lines. Solving this in a specific instance gives the two original 2-flats, which likewise implies that the frame-convex hull of two general 2 -flats in $\mathbb{R}^{4}$ consists only of the original 2-flats.

\section{REFERENCES}

[1] F. Ardila and C. Klivans, The Bergman complex of a matroid and phylogenetic trees. arXiv: math.CO/0311370.

[2] L. Danzer, B. Grünbaum, and V. Klee, Helly's theorem and its relatives, in V. Klee, ed., Convexity, Proc. Symp. Pure Math., Vol. 7, Amer. Math. Soc., Providence, 1963, 100-181.

[3] J. Eckhoff, Helly, Radon, and Carathéodory type theorems, in P.M. Gruber and J.M. Wills, eds., Handbook of Convexity, Vol. A, North-Holland, Amsterdam, 1993, 389-448.

[4] W. Fulton, Intersection theory, Ergebnisse der Math., no. 2, Springer-Verlag, 1998, second edition.

[5] J. E. Goodman and R. Pollack, Foundations of a theory of convexity on affine Grassmann manifolds, Mathematika 42 (1995), 305-328. MR 97a:52002

[6] J. E. Goodman, R. Pollack, and R. Wenger, Geometric transversal theory, in J. Pach, ed., New Trends in Discrete and Computational Geometry, Springer-Verlag, Berlin, 1933, 163-198. 
[7] P. Griffiths and J. Harris, Principles of algebraic geometry, J. Wiley and Sons, 1978.

[8] B. Grünbaum, On common transversals, Arch. Math. 9 (1958), 465-469. MR 21 \#1566

[9] J. Harris, Algebraic geometry: A first course, Graduate Texts in Mathematics 133, Springer-Verlag, 1992.

[10] E. Helly, Über Mengen konvexer Körper mit gemeinschaftlichen Punkten, Deutsch. Math. Verein 32 (1923), $175-176$.

[11] M. M. Kapranov, Chow quotients of Grassmannians. I, I. M. Gel'fand Seminar, Adv. Soviet Math., vol. 16, Amer. Math. Soc., Providence, RI, 1993, pp. 29-110. MR 95g:14053

[12] J. Radon, Mengen konvexer Körper, die einen gemeinsamen Punkt enthalten, Math. Ann. 83 (1921), $113-115$.

[13] L. Santaló, Un theorema sobre conjuntos de paralelepipedos de aristas paralelas, Publ. Inst. Mat. Univ. Nac. Litoral 2 (1940), 49-60. MR 2, $261 \mathrm{f}$

[14] David Speyer and Bernd Sturmfels, The Tropical Grassmannian. arXiv: math.AG/0304218

[15] P. Vincensini, Figures convexes et variétés linéaires de l'espace euclidien a $n$ dimensions, Bull. Sci. Math. (2), 59 (1935), 163-174.

[16] R. Wenger, Progress in geometric transversal theory, in B. Chazelle, J. E. Goodman, and R. Pollack, eds., Advances in Discrete and Computational Geometry, Vol. 223, Contemp. Math., Amer. Math. Soc., Providence, 1999.

[17] R. Wenger, Helly-type theorems and geometric transversals, in J. E. Goodman and J. O'Rourke, eds., Handbook of Discrete and Computational Geometry, 2nd ed., CRC Press, Boca Raton, 2004, pp. 73-96.

Department of Mathematics, City College (CUNY), New York, NY 10031, USA

E-mail address: jegcc@cunyvm.cuny.edu

$U R L:$ http://www.ccny. cuny.edu/mathematics/faculty/faculty.htm

Matematisk institutt, Universitetet i Bergen, Johannes Bruns gt. 12, 5008 Bergen, NorWAY

E-mail address: andreash@mi.uib.no

URL: http://www.mi.uib.no/ ªndreash/index.html

Department of Mathematics, Courant Institute of Mathematical Sciences, New York University, 251 Mercer St., New York, NY 10012, USA

E-mail address: pollack@cims.nyu.edu

URL: http://www.math.nyu.edu/faculty/pollack/

Matematisk institutt, Universitetet i Oslo, PO Box 1053, Blindern, NO-0316 Oslo, NorWAY

E-mail address: ranestad@math.uio.no

$U R L:$ http://www.math.uio.no/ ranestad

Department of Mathematics, Texas A\&M University, College Station, TX 77843, USA

E-mail address: sottile@math.tamu.edu

$U R L:$ http://www . math. tamu.edu/ ${ }^{\sim}$ sottile 